\title{
Dashboard to evaluate student engagement with interactive simulations
}

\author{
Diana López-Tavares, ${ }^{1}$ Katherine Perkins, ${ }^{2}$ Sam Reid, ${ }^{2}$ Michael Kauzmann, ${ }^{2}$ and Carlos Aguirre-Vélez ${ }^{1}$ \\ ${ }^{1}$ CICATA-Legaria, Instituto Politécnico Nacional, Mexico City, MX 07320 \\ ${ }^{2}$ Physics Department, University of Colorado, Boulder, CO, 80309
}

\begin{abstract}
Student engagement with interactive simulations is affected by the prompts and activities teachers choose to couple with such simulations. In this early work, we introduce a prototype dashboard to visualize and evaluate student engagement generated by activities that leverage interactive simulations. Engagement in this study is gauged by factors such as the time spent in the activity, the rate and pattern of clicks, and the simulation elements used by students. The dashboard employs several approaches to visualize student mouse activity data, displaying either individual student interactions or aggregated information of an entire group. To test the dashboard's usability, we evaluate student engagement resulting from a homework activity that focused on factors that affect stored energy in a capacitor and used the PhET simulation "Capacitor Lab: Basics". The results show that the dashboard's visualizations help provide a general idea of how students interact, describe their engagement, and are a promising tool for guiding activity design.
\end{abstract}

\section{INTRODUCTION}

Some research studies on learning have revealed connections between student engagement and learning results [1]. For PhET Interactive Simulations, a project that develops interactive simulations (sims) for science and math, student engagement with their sims is important to addressing science practice goals, e.g. exploring, generating questions, making predictions, testing ideas, designing experiments, and monitoring one's own understanding [1].

Studies show a connection between the level and type of guidance used in an activity and student engagement with PhET sims [1-4]. The results show that appropriate guidance can help focus students on sim elements that are important for achieving specific learning goals. However, excessive guidance can lead students to follow directions rather than engaging in the deep exploration associated with the science practice goals.

Designing activities that strike the optimal balance between generating engagement and scaffolding for specific learning goals can be challenging for teachers. In environments where the teacher cannot directly observe the student interactions (e.g. homework), the challenge is even greater. In this paper, we present the design and test of a prototype dashboard to help stakeholders characterize student engagement in PhET sim-centered activities.

A dashboard displays important information in visual representations. The use of dashboards in education is not new, but currently all such dashboards are focused on Learning Management Systems (LMS), Massive Open Online Courses (MOOCs), and Intelligent Tutor Systems (ITS) $[8,9]$. These educational systems use well defined metrics to generate their visualizations, such as student logins, question tries/correctness, material viewing history, and more. Student data is often binary (correct/incorrect) and linear. In the case of interactive simulations, these are openended environments where each student can manipulate them in many complicated ways. The data produced by such interactions is quite different from other education products and a different approach to data sources, analysis and visualizations is needed.

In this research, student engagement is assumed to be correlated with their exploration of the sim: the duration; number, location, and evolution of clicks; and interactive elements used.

We emphasize that the intent of this work is to represent engagement, not learning. The dashboard may show that students interact (or not) with certain sim elements, but this information does not necessarily imply that students are understanding or learning.

The dashboard visualizations are intended to be a useful tool for teachers and instructional designers. Knowing what students may have seen or done in the sim could help generate class discussion or aid in improving activity design. In this early work, we present the design of the dashboard visualizations. We also test its potential to describe students' engagement by examining interaction data collected from a homework activity that use the PhET sim Capacitor Lab: Basics.

\section{METHODOLGY}

\section{A. Data sources}

PhET-iO is an extension of the PhET project that allows all student-sim interactions to be captured in a data stream file. Informed by prior work related to engagement with PhET sims $[1,2,4]$, we selected the following data for use in the dashboard design:

- GroupID and userID (to parse by group and individual user).

- Events and its position. The position of clicks and where drag movements start (mouse-up) and end (mouse- 
down) are captured.

- Total time and time between events.

- $\quad$ Record of sim elements used.

\section{B. Sim Section}

PhET Interactive Simulations have a wide variation of manipulation complexity. For this prototype dashboard, the sim selected should have enough content to test engagement but reasonably constrained interaction. We selected an adaptation of Capacitor Lab: Basics. In this sim, students can explore the physics of a parallel plate capacitor. Students can change the plate area, the separation distance, the connection to the battery and the battery voltage. Students can see charges build up on the plates, graphs of capacitance, stored energy, and plate charge, and an electric field representation. A voltmeter is also available.

\section{Indicators and visualizations}

Some indicators and visualizations to identify engagement were adapted from prior work [2,4], for instance, the elements in the sim used, total time of interaction, and total events and rate of events/min, as well as specific visualizations used in Google Analytics to understand website use. Figure 1 shows the current elements of the dashboard design.

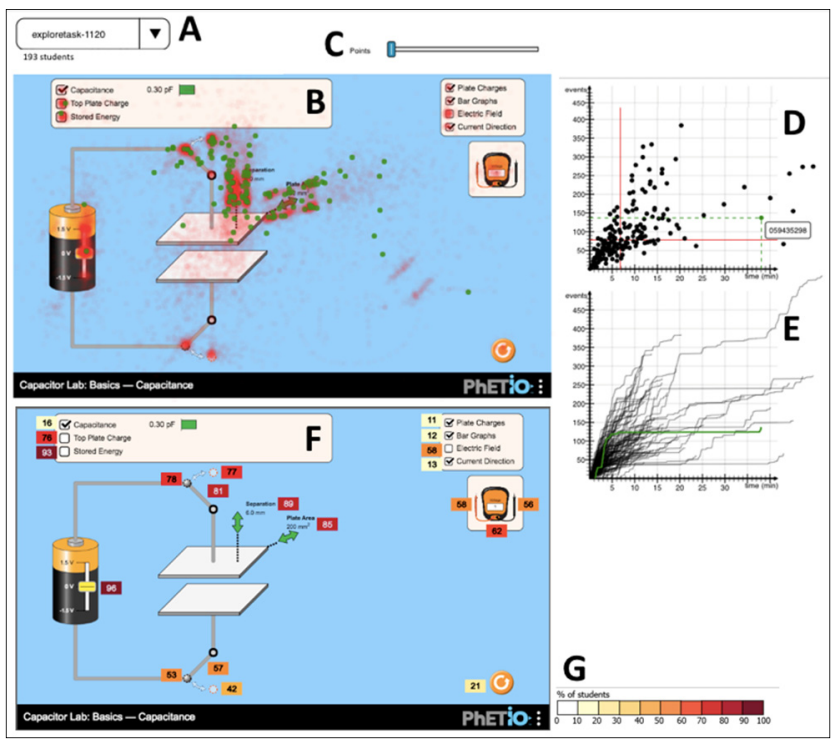

FIG 1. Dashboard design. The user can select the group to visualize (A), see the location of the events of the group or one student (B), change the opacity of dots in the events map (C), see the total events, total time of use, and time evolution of interactions of each student (D and E), and see the percentage of students that use each element of the sim (F) using a color code to identify the more used elements

1 The dashboard prototype can be explored in https://bit.ly/2IwwhuD with the group name exploretask-1120.
Dashboard users can select the group they want to analyze (Fig. 1-A). The main visualization is the events map (Fig. 1-B) where red dots represent the location of all the events (clicks, mouse-down and mouse-up) of the group overlaid on a sim screenshot. The dot opacity can be modified (Fig. 1-C) to optimize the visualization for groups with different number of students.

In Fig. 1-D each dot represents one student, the horizontal coordinate represents the total time of interaction and the vertical coordinate represents the total number of events. The red lines in the graph are the median values of total time and events for that group. The user can select individual students by clicking dots in this graph. The color of the dots selected changes from black to a distinct color and the events of the selected student then appears in the events map with this same distinct color over the red dots. Thus, the individual student can easily be compared with the information of the group (Fig. 1-B). In addition, the line representing the chosen student in the graph displaying time evolution of interaction (Fig. 1-E) also changes to match this distinct color. This graph helps to visualize how the rate of events/min evolved during the manipulation of the sim. Several students can be selected from the dots graph simultaneously.

The elements used map (Fig. 1-F) is a screenshot of the sim with colored boxes displaying the percentage of students that used each interactive element. The color of the boxes corresponds to a color gradient indicating usage (Fig. 1-G).

\section{Testing}

Sim interaction data generated from a homework activity was collected to test the design of the dashboard and its ability to describe student engagement. A group of 193 university students in an electromagnetics course were asked to "explain how changing each factor in the simulation changes the amount of stored energy when the capacitor is connected to the battery and when it is disconnected".

The activity was assigned just before a lecture about capacitors and capacitance. The homework answers, beyond the interaction data, are not considered in this analysis.

\section{RESULTS}

Using the dashboard visualizations ${ }^{1}$, we describe the student engagement generated by the homework activity. We analyze the information in the maps (events and elements used) and the time evolution of interaction.

\section{A. Maps of Events and Elements Used}

Figure 2 shows the events and elements-used maps displaying data from the homework activity. We can immediately see the most used elements are the battery and 


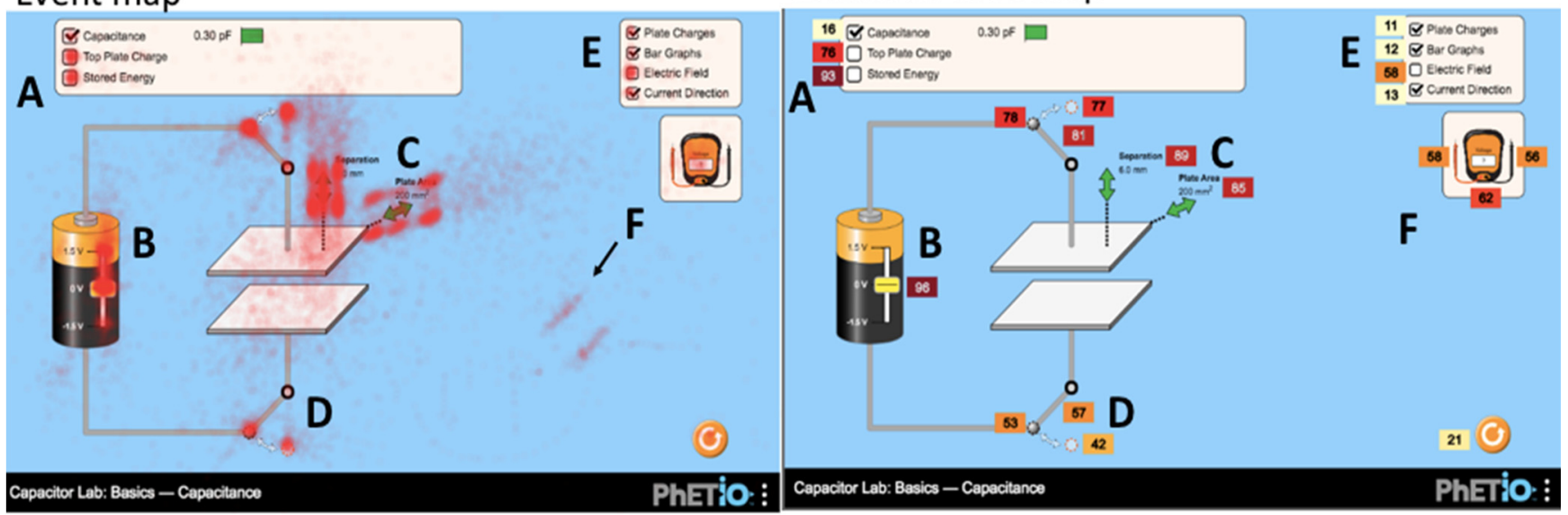

FIG 2. Visualizations in the dashboard results of the homework about stored energy. Events map shows the pattern of events with a bigger concentration in the battery and the Plates separation (B and $\mathrm{C}$ ). Elements used map shows in dark red the elements more used in the activity (A, B and C), and in light colors the elements less used (E).

the controls for plate area and separation. A more detailed analysis provides more insights (matching letters in Fig. 2):

A. The activity requires the activation of the Stored Energy checkbox to see the change of this variable, yet $7 \%$ of the students did not activate this element.

B. While most students (96\%) change the battery voltage, the concentration of click events are at $0 \mathrm{~V}$ and $1.5 \mathrm{~V}$, the maximum value. The pattern of events shows students tested some other positive voltage values, but only a few students tested negative voltage values.

C. Slightly less than $90 \%$ of the students modify the area and separation of the plates. These two elements are related. If one of them is modified, the position of the other control element changes. In the events map we can observe three parallel shapes of lines related to the control that changes the separation of the plates (vertical arrow in the screenshot). The left line pattern of events is created when the area of the plates is minimum, the middle one corresponds to the default area value when the sim starts or is refreshed, and the line on the right occurs when the plate area is maximum. We observe a greater concentration of events at the extremes (min and max values for the separation) in the three cases, but also a significant number of events in intermediate values. This information suggests that several students tested different values of this variable with some combination of plate area values. On the other side, the plate area control has its concentration of events in the extremes and only two parallels shapes of lines. This pattern suggests that with the default value of separation, most of the students didn't change the plate area value, and different values of this variable were generally not tested.

D. The activity asks the student to connect and disconnect the capacitor from the battery. In the sim this action can be accomplished with switches at the top or bottom of the circuit. It is difficult to say what percentage of students completed this aspect of the homework. However, the dashboard shows the top switch to be the more used element. This information is useful for sim designers.

E. Both maps show that the elements active by default in the sim (checkboxes selected) have low interaction. This information can be useful for instructional designers to know that students commonly leave these selected.

F. The voltmeter has low interaction $(62 \%$ students touch it and less that $60 \%$ use the probes). The parallel lines of red dots in the $\mathrm{F}$ spot in the events map show the positions of the probes when the voltmeter is taken out of the tool box. This evidence suggests that students move the probes, however we cannot currently predict the positions to which the probes were moved using the events map. It is possible to display more information in the dashboard related, originally the events map included lines that represented drag movements. However, with almost 200 students, the saturation of information was overwhelming and difficult to interpret to how the sim was used. How to represent drag movements remains an open question.

\section{B. Time evolution of interactions}

Figure 3 shows graphs of events versus time for individual students. Each dot in the Total events vs time graph (left graph in Fig. 3) represents one student. We can see the distribution of the dots, with a high density of dots in the time interval 0-7 minutes. The median value for time spent on this activity is 6.8 minutes and for total number of events is 79 for this group (marked with red lines).

To examine specific student interaction, we select four representative behaviors in Fig. 3 - Students A, B, C, and D.

Student $\mathrm{A}$ is one with a small number of events and time interaction (around 60 events in 2 minutes). According to 


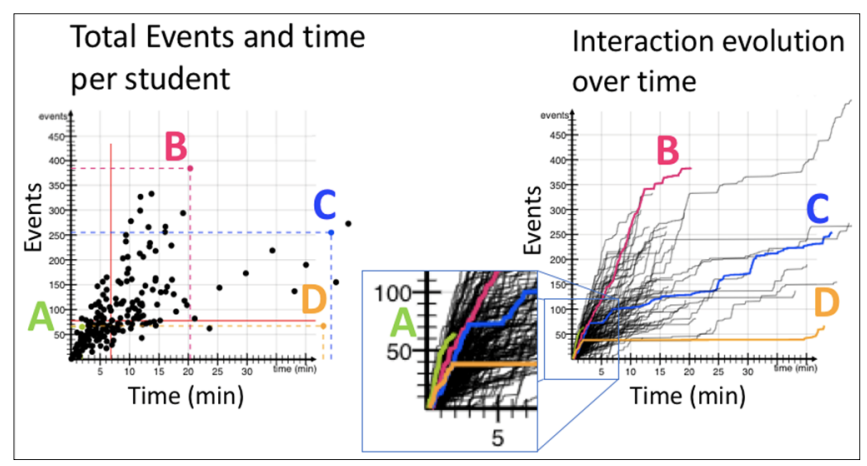

FIG 3. Events vs Time graphs, showing four students with different patterns (A-D) of interaction and engagement.

PhET interview data with the sim [1], in the first 2-5 minutes of interaction (depending on the sim), students are generally "playing around" discovering the sim's functions. As such, in the first minutes most students show a large slope curve in the evolution graph (many clicks in a small amount of time). Student A is considered to have initially high, but unsustained, engagement.

Other research suggests that short pauses in interaction is often time spent by the student to take notes, reflect, or analyze what is happening in the $\operatorname{sim}[3,8]$. For student $B$, we observe over 10 minutes of very active interaction with small time between interactions (steep slope in the curve), but after that, we see almost 10 minutes with slowed interaction. This suggests a more planned manipulation or potentially a time moving between interaction and answering the homework. Student $\mathrm{C}$ has an interaction evolution that suggests a more analyzed manipulation for the time between one event and other one (medium slope). Both students are considered to demonstrate high engagement.

Student D has a large interval of time of not interacting with the sim (more than 30 minutes). Likely, this student left the homework page open with the sim while working on another task. We can observe several students with some long horizontal lines. We use median, as opposed to mean, values for group characteristics to minimize the influence of these outliers. Student D is considered displaying low engagement.

\section{CONCLUSIONS AND FUTURE WORK}

This prototype dashboard is successfully capturing aspects of student interaction and engagement with the open exploratory environment of a simulations. The visualization shows overall patterns of interaction - e.g. in most cases students interacted with the extreme values of interactive elements and some tools were not used by nearly half of the students (electric field and the voltmeter). This type of information can inform activity design. If more interaction with these elements is desired for learning, the activity should be modified to promote their use.

The dashboard also shows the pattern of interaction is different for each student, which aligns with the expectation of guided inquiry activities and provides opportunity for teachers to examine individual student engagement. While not feasible in large lecture classes, teachers in smaller classes may value this capability to review any students' engagement on activities performed outside of class. In the test activity, there were a large number of students with less than 5 minutes of interaction, below the target level of engagement. One explanation is that this homework activity was added on as the last problem in the normal homework and may have been deemed "not worth the time" by students.

We are working to improve the dashboard design with a section that allows comparison of engagement across different groups. We are also considering providing information about the elements used by each student, about drag movements, and about the average distribution of an interactive element's state during sim use.

To further test, inform, and advance the ability of the dashboard to characterize engagement, we plan to collect and analyze data from diverse activities and a diverse set of sims.

\section{ACKNOWLEDGEMENTS}

The authors would like to acknowledge the whole PhET team for their support, and the generous funding provided by CONACYT (Mexico), the Gordon and Betty Moore Foundation, and the University of Colorado Boulder.
[1] N. S. Podolefsky, K. K. Perkins, and W. K. Adams, Phys. Rev. Spec. Top. - Phys. Educ. Res. 6, (2010).

[2] J. M. Chamberlain, K. Lancaster, R. Parson, and K. K. Perkins, Chem. Educ. Res. Pr. Chem. Educ. Res. Pr. 15, 628 (2014).

[3] W. K. Adams, A. Paulson, and C. E. Wieman, in AIP Conf. Proc. (2008), pp. 59-62.

[4] E. B. Moore, T. A. Herzog, and K. K. Perkins, Chem. Educ. Res. Pr. 14, 257 (2013).

[5] K. Verbert, S. Govaerts, E. Duval, J. Santos, F. Assche, G. Parra, and J. Klerkx, Pers. Ubiquitous Comput. 1
(2013)

[6] B. A. Schwendimann, M. J. Rodríguez-Triana, A. Vozniuk, L. P. Prieto, M. Shirvani Boroujeni, A. Holzer, D. Gillet, and P. Dillenbourg, IEEE Trans. Learn. Technol. 10, 148 (2017).

[7] E. B. Moore, Y.-Y. Carpenter, R. Parson, and K. Perkins, 1 (2014).

[8] S. Perez, J. Massey-allard, D. Butler, J. Ives, D. Bonn, N. Yee, and I. Roll, Proc. Artif. Intell. Educ. 10331, 287 (2017). 